\title{
Deltafunction Model for the Helium Dimer
}

\author{
LAWRENCE L. LOHR AND S. M. BLINDER \\ Department of Chemistry, University of Michigan, Ann Arbor, Michigan 48109-1055
}

Received April 19, 1994; accepted August 10, 1994

\begin{abstract}
The helium dimer ${ }^{4} \mathrm{He}_{2}$ has recently been detected, confirming earlier ab initio predictions of stability for a single bound state with binding energy of $1.310 \mathrm{mK}$. The predicted potential minimum is at $2.96 \AA$, with a radial distribution function peaking at $6.96 \AA$. We model this system using a Dirac bubble potential, which also admits just one bound $J=0$ state. With the bubble located at $6.96 \AA$, an overlap of 0.9994 with the ab initio wave function is obtained. An average internuclear distance of $52.6 \AA$ is calculated, in good agreement with the $a b$ initio result. The root mean square deviation from the mean, $48.0 \AA$, indicates an enormous spread of the radial wave function. Also consistent with our model is the absence of bound states for the isotopic variants ${ }^{3} \mathrm{He}^{4} \mathrm{He}$ and ${ }^{3} \mathrm{He}_{2}$. Cross sections for helium-helium scattering are also computed, using both a partial-wave expansion and the Born approximation. General trends in the energy dependence of the total cross section are accounted for, in qualitative agreement with experimental results. (c) 1995 John Wiley \& Sons, Inc.
\end{abstract}

\section{Introduction}

$\mathbf{T}$ he long-sought ${ }^{4} \mathrm{He}_{2}$ gaseous diatomic molecule has recently been detected in extreme pulsed expansions of He [1]. Stability of a single bound state with $v=0, J=0$ had been expected, based on highly accurate computations of the interaction between two ${ }^{4} \mathrm{He}$ atoms. No other states bound with respect to dissociation are predicted. For example, the "LM2M2" analytic representation of the potential $[2,3]$ is characterized by a well depth of $10.970 \mathrm{~K}$, a binding energy of only $1.310 \mathrm{mK}$ for the $v=0, J=0$ state, and an equilibrium internuclear distance of $5.61 \mathrm{bohr}$
$(2.97 \AA)$. This potential was constructed by fitting both $\mathrm{ab}$ initio calculated points $[4,5]$ and experimental data on viscosity and the second virial coefficient. A recent quantum Monte Carlo calculation [6] yielded a well depth of $11.01 \pm 0.10 \mathrm{~K}$ and an equilibrium separation of 5.6 bohr $(2.96 \AA)$. The marginal stability of this molecule is reflected in the extremely large mean internuclear separation, $\langle r\rangle=98.1$ bohr $(51.9 \AA)$ as calculated [7] from the wave function associated with the LM2M2 potential [2]. In addition, it has been predicted [8] that no bound states exist for either of the isotopic variants ${ }^{3} \mathrm{He}^{4} \mathrm{He}$ or ${ }^{3} \mathrm{He}_{2}$. It has recently been shown [7] that the effect of retardation is to decrease the binding energy of ${ }^{4} \mathrm{He}_{2}$ by about $10 \%$ and to increase $\langle r\rangle$ by 
about $5 \%$. A number of other theoretical treatments of the $\mathrm{He}-\mathrm{He}$ interaction have been presented [9].

\section{Model}

We propose, as a simple model for the interaction between two helium atoms, an attractive deltafunction potential in the shape of a spherical bubble [10]. This "Dirac bubble potential" had previously been applied to model the Fermi contact interaction [11] and the photodetachment of an electron from the $\mathrm{C}_{60}$ molecule [12]. Our model for $\mathrm{He}_{2}$ requires just two parameters: the equilibrium separation $r_{0}$ and the effective strength of the potential, as represented by the constant $\alpha$.

A deltafunction model might appear, at first glance, to be an inappropriate representation for a potential energy function so broad and shallow. However, several key features of the single bound state of the actual potential lend support to our model. The computed $[6,7]$ mean internuclear separation of $51.9 \AA$ is very large compared to the equilibrium separation of $2.96 \AA$ and to the outer turning radius of $14.09 \AA$, indicating that the "particle" spends much of its time very far from its equilibrium position. Thus, the potential well is actually narrow on the scale of its mean separation. The Dirac deltafunction represents the limiting case of a narrow potential, the concomitant infinite depth being just incidental. It should be noted that the strength of the model potential is determined, not by its depth, but by the multiplicative parameter $\alpha$ that is fitted to the ab initio binding energy. As $\alpha$ approaches negative infinity, the number of bound states increases and the model becomes equivalent to a rigid rotor.

The Schrödinger equation for a diatomic species bound by a spherical deltafunction potential can be written

$$
\left[-\frac{\hbar^{2}}{2 \mu} \nabla^{2}+\frac{\alpha}{4 \pi r_{0}^{2}} \delta\left(r-r_{0}\right)\right] \psi(\mathbf{r})=E \psi(\mathbf{r}) .
$$

Spherical symmetry allows the factorization $\psi(\mathbf{r})=$ $R_{\kappa J}(r) Y_{J M}(\theta, \phi)$. The radial equation for the bound $J=0$ level can be written

$$
\begin{aligned}
{\left[-\kappa^{2}+\frac{1}{r^{2}} \frac{d}{d r} r^{2} \frac{d}{d r}\right] R_{\kappa}(r)=} & \left(\lambda / r_{0}\right) \\
& \times \delta\left(r-r_{0}\right) R_{\kappa}\left(r_{0}\right),
\end{aligned}
$$

having defined

$$
E \equiv-\hbar^{2} \kappa^{2} / 2 \mu
$$

and

$$
\lambda \equiv \mu \alpha / 2 \pi \hbar^{2} r_{0} .
$$

Equation (2) has been solved exactly [10] by exploiting its isomorphism with free-particle partialwave Green's functions. For $\lambda<0$, corresponding to an attractive deltafunction potential, there exists one and only one bound state for each angular momentum $J$ for which the condition

$$
|\lambda|>2 J+1
$$

is fulfilled. The normalized $J=0$ eigenfunction is given by

$$
\begin{aligned}
R_{\kappa}(r)= & {\left[\frac{1-\left(2 \kappa r_{0}+1\right) e^{-2 \kappa r_{0}}}{4 \kappa^{5} r_{0}^{2}}\right]^{-1 / 2} } \\
& \times \frac{\sinh \left(\kappa r_{<}\right)}{\kappa r_{<}} \frac{e^{-\kappa r_{>}}}{\kappa r_{>}},
\end{aligned}
$$

where $r>$ and $r<$ are, respectively, the larger and smaller of $r, r_{0}$. The corresponding eigenvalue is determined by the connection at $r=r_{0}$, viz.,

$$
\frac{e^{-2 \kappa r_{0}}-1}{2 \kappa r_{0}}=\frac{1}{\lambda} \text {. }
$$

The reported binding energy, $1.310 \mathrm{mK}$, corresponds to $E=-4.148 \times 10^{-9}$ Hartrees. We find [cf. Eq. (3)] $\kappa=0.005520$ bohr $^{-1}$. The most obvious choice for $r_{0}$ in Eqs. (6) and (7) is the radius at the potential minimum, $2.9596 \mathrm{~A}$ or $5.61 \mathrm{bohr}$. With this value, the overlap integral between the wave function (6) and that obtained from the LM2M2 potential equals 0.95 . If we choose, instead, $r_{0}=$ 13.15 bohr $(6.96 \AA)$, corresponding to the maximum of the LM2M2 radial distribution function, we obtain the amazing overlap.

$$
\int_{0}^{\infty} R_{\text {bubble }}(r) R_{\mathrm{LM} 2 \mathrm{M} 2}(r) r^{2} d r=0.99942 .
$$

We will therefore adopt the value $r_{0}=13.15 \mathrm{bohr}$ in our subsequent computations.

Two views of the radial distribution function $D(r)=r^{2} R^{2}(r)$ are plotted in Figures 1 and 2, with the LM2M2 function shown dashed. As in all solutions of the bubble potential, a cusp occurs at $r=r_{0}$. The extreme delocalization of the wave function reflects a value of $\kappa$ very nearly zero.

From Eq. (7), we identify $\lambda_{4-4}=-1.0741$, just barely fulfilling condition (5) for a $J=0$ bound 


\section{$\mathbf{D}(\mathbf{r})$}

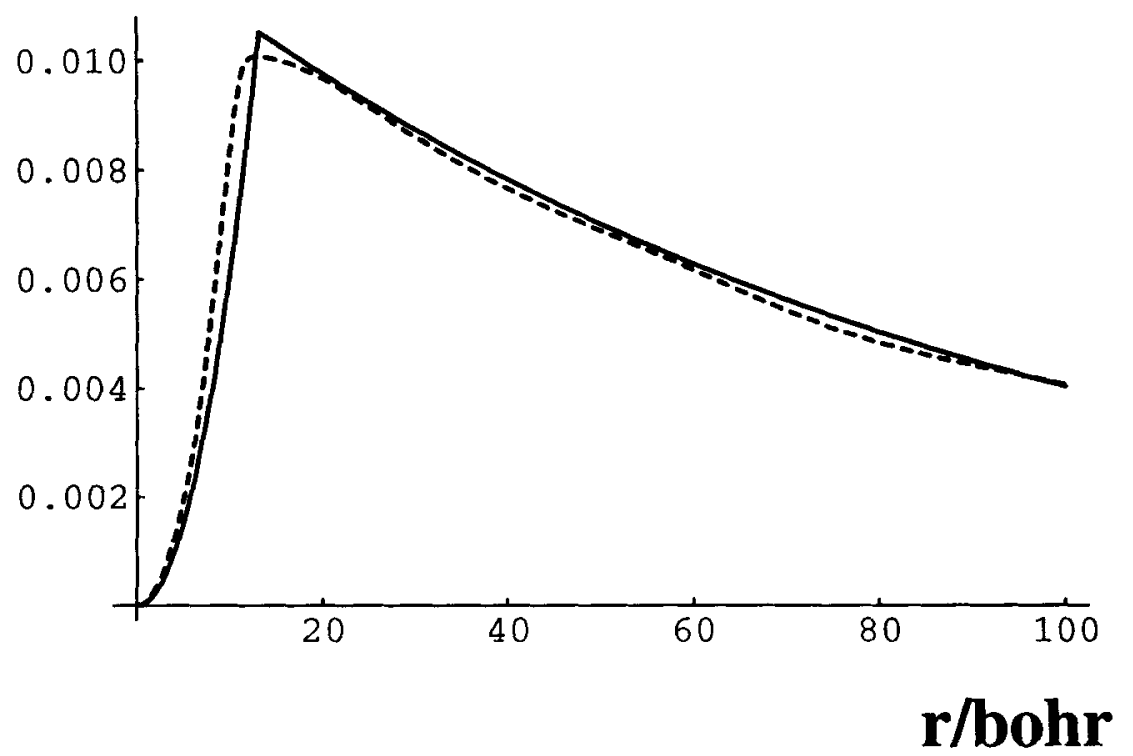

FIGURE 1. Radial distribution function $D(r)$ for the $J=0$ bound state of ${ }^{4} \mathrm{He}_{2}$. Solid line: bubble potential; dashed line: LM2M2 computation.

state. If we assume that the parameter $\alpha$ in Eqs. (1) and (4) characterizes the $\mathrm{He}-\mathrm{He}$ potential function, then $\lambda$ is proportional to the reduced mass $\mu$. This implies, for ${ }^{3} \mathrm{He}^{4} \mathrm{He}, \lambda_{3-4}=-0.9207$, and for ${ }^{3} \mathrm{He}_{2}, \lambda_{3-3}=-0.8056$, thus no bound state for either isotopic variant.
The expectation value of $r$ for the wave function (6) works out to

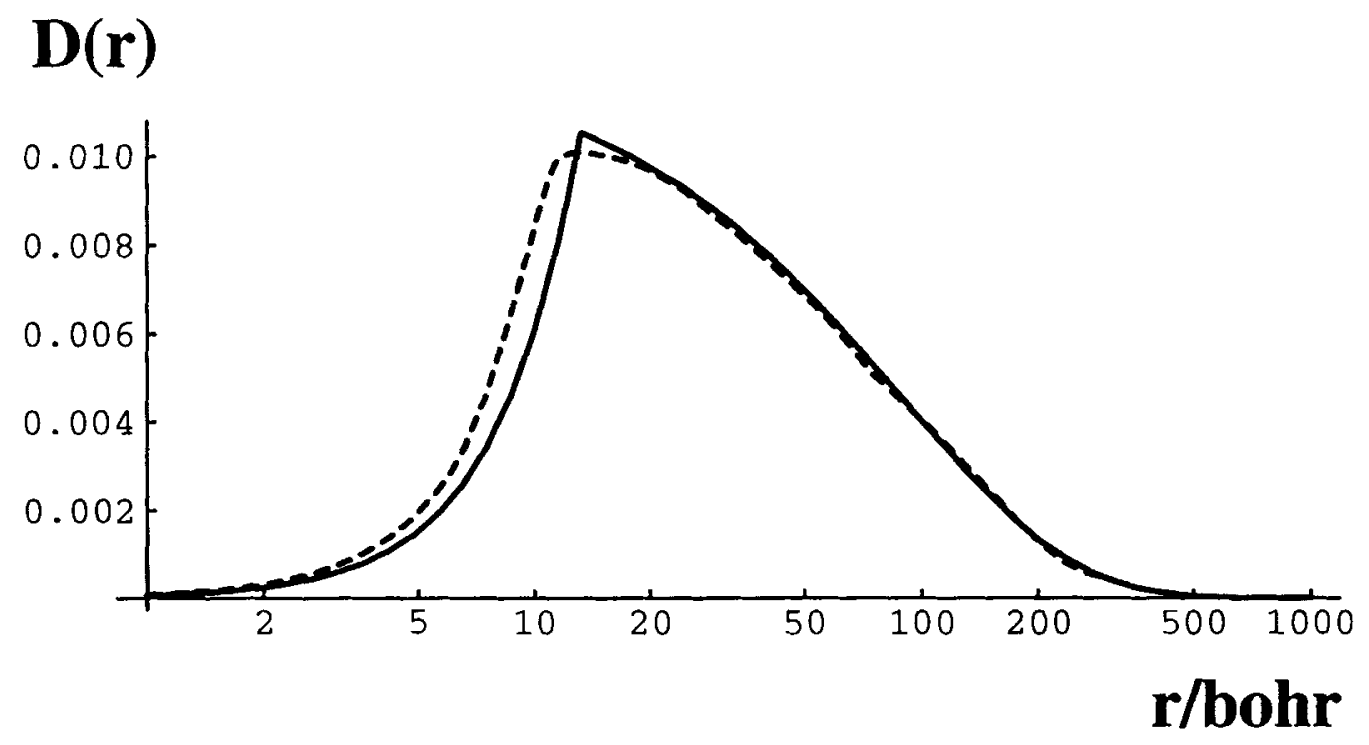

FIGURE 2. Radial distribution function $D(r)$ vs. $r$ on logarithmic scale. Solid line: bubble potential; dashed line: LM2M2 computation. 
With the chosen parameters, $\langle r\rangle=99.4 \mathrm{bohr}$ or $52.6 \AA$, agreeing well with the ab initio value: $51.9 \AA$. The rms deviation from the mean equals 90.6 bohr or $48.0 \AA$.

The bubble-potential model also enables calculation of scattering cross sections for the three pairs ${ }^{4} \mathrm{He}-{ }^{4} \mathrm{He},{ }^{3} \mathrm{He}-{ }^{3} \mathrm{He}$, and ${ }^{3} \mathrm{He}-{ }^{4} \mathrm{He}$ (abbreviated $4-4,3-3$, and $3-4$, respectively). The continuum eigenfunctions can be represented as follows:

$$
\begin{aligned}
R_{k, l}(r)= & (2 k / \pi)^{1 / 2} \\
& \times\left[j_{l}\left(k r_{>}\right) \cos \delta_{l}-y_{l}\left(k r_{>}\right) \sin \delta_{l}\right] \\
& \times j_{l}\left(k r_{<}\right) / j_{l}\left(k r_{0}\right),
\end{aligned}
$$

where $j_{l}$ and $y_{l}$ are spherical Bessel functions and

$$
E=\hbar^{2} k^{2} / 2 \mu \text {. }
$$

The phase shifts $\delta_{l}$ are determined by

$$
\cot \delta_{l}=\frac{y_{l}\left(k r_{0}\right)}{j_{l}\left(k r_{0}\right)}-\frac{1}{\lambda k r_{0}\left[j_{l}\left(k r_{0}\right)\right]^{2}} .
$$

For ${ }^{3} \mathrm{He}^{4} \mathrm{He}$, the scattering amplitude is given by

$$
f(\theta)=(2 i k)^{-1} \sum_{l=0}^{\infty}(2 l+1)\left(e^{2 i \delta_{l}}-1\right) P_{l}(\cos \theta) .
$$

This gives the differential cross section

$$
\begin{aligned}
\sigma(\theta) & =|f(\theta)|^{2} \\
& =\frac{1}{k^{2}}\left|\sum_{l=0}^{\infty}(2 l+1) e^{i \delta_{l}} \sin \delta_{l} P_{l}(\cos \theta)\right|^{2}
\end{aligned}
$$

and the total scattering cross section

$$
\begin{aligned}
\sigma_{\text {tot }}^{3-4} & =2 \pi \int_{0}^{\pi}|f(\theta)|^{2} \sin \theta d \theta \\
& =\frac{4 \pi}{k^{2}} \sum_{l=0}^{\infty}(2 l+1) \sin ^{2} \delta_{l} .
\end{aligned}
$$

For ${ }^{4} \mathrm{He}-{ }^{4} \mathrm{He}$, the scattering amplitude must be replaced by the symmetric combination $f(\theta)+$ $f(\pi-0)$, to take account of boson statistics. Thus, only the even-l partial waves contribute with

$$
\sigma_{\mathrm{tot}}^{4-4}=4 \times \frac{4 \pi}{k^{2}} \sum_{l=0,2 \ldots}^{\infty}(2 l+1) \sin ^{2} \delta_{l} .
$$

For scattering of the fermions ${ }^{3} \mathrm{He}-{ }^{3} \mathrm{He}$, even partial waves contribute for the singlet nuclearspin state and odd partial waves for the triplet.
Assuming a 1:3 statistical population ratio, the total cross section works out to

$$
\begin{aligned}
\sigma_{\mathrm{tot}}^{3-3} & =\frac{4 \pi}{k^{2}} \\
& \times\left[\sum_{l=0,2 \ldots}^{\infty}(2 l+1) \sin ^{2} \delta_{l}+3 \sum_{l=1,3}^{\infty}(2 l+1) \sin ^{2} \delta_{l}\right] .
\end{aligned}
$$

Figure 3 shows the energy dependence of the total cross section for $\mathrm{He}-\mathrm{He}$ scattering. Although these results do not agree quantitatively with experimental data [13], they do give the correct trends. Thus, the $4-4$ curve dips around $1 / 2 \mathrm{meV}$ to reflect the missing $l=1$ partial wave. The 3-3 curve shows enhancement of odd- $l$ partial waves compared to the 3-4.

For scattering at higher energies, the Born approximation can be applied. For a spherically symmetric potential

$$
\begin{aligned}
f(\theta) & =-\frac{2 \mu}{\hbar^{2} K} \int_{0}^{\infty} r \sin (K r) V(r) d r, \\
K & \equiv 2 k \sin \frac{\theta}{2} .
\end{aligned}
$$

For the bubble potential,

$$
V(r)=\frac{\alpha}{4 \pi r_{0}^{2}} \delta\left(r-r_{0}\right) ;
$$

thus

$$
f(\theta)=-\lambda \frac{\sin \left(K r_{0}\right)}{K} .
$$

For helium 3-4 scattering, the total cross section is given by

$$
\begin{aligned}
\sigma_{\text {tot }}^{3-4} & =2 \pi \int_{0}^{\pi}|f(\theta)|^{2} \sin \theta d \theta \\
& =\frac{2 \pi \lambda^{2}}{k^{2}} \int_{0}^{2 k} \frac{\sin ^{2}\left(K r_{0}\right)}{K} d K \\
& =4 \pi r_{0}^{2} \lambda^{2} F\left(2 k r_{0}\right),
\end{aligned}
$$

where

$$
F(x) \equiv[\gamma+\ln (2 x)-\mathrm{Ci}(2 x)] / x^{2} .
$$

Here, $\gamma$ is Euler's constant $(0.5772 \cdots)$, and $\mathrm{C}$, the cosine integral:

$$
\mathrm{Ci}(z) \equiv-\int_{z}^{\infty} \frac{\cos t}{t} d t .
$$

For helium 4-4 scattering,

$$
\sigma_{\text {tot }}^{4-4}=2 \pi \int_{0}^{\pi}|f(\theta)+f(\pi-\theta)|^{2} \sin \theta d \theta .
$$



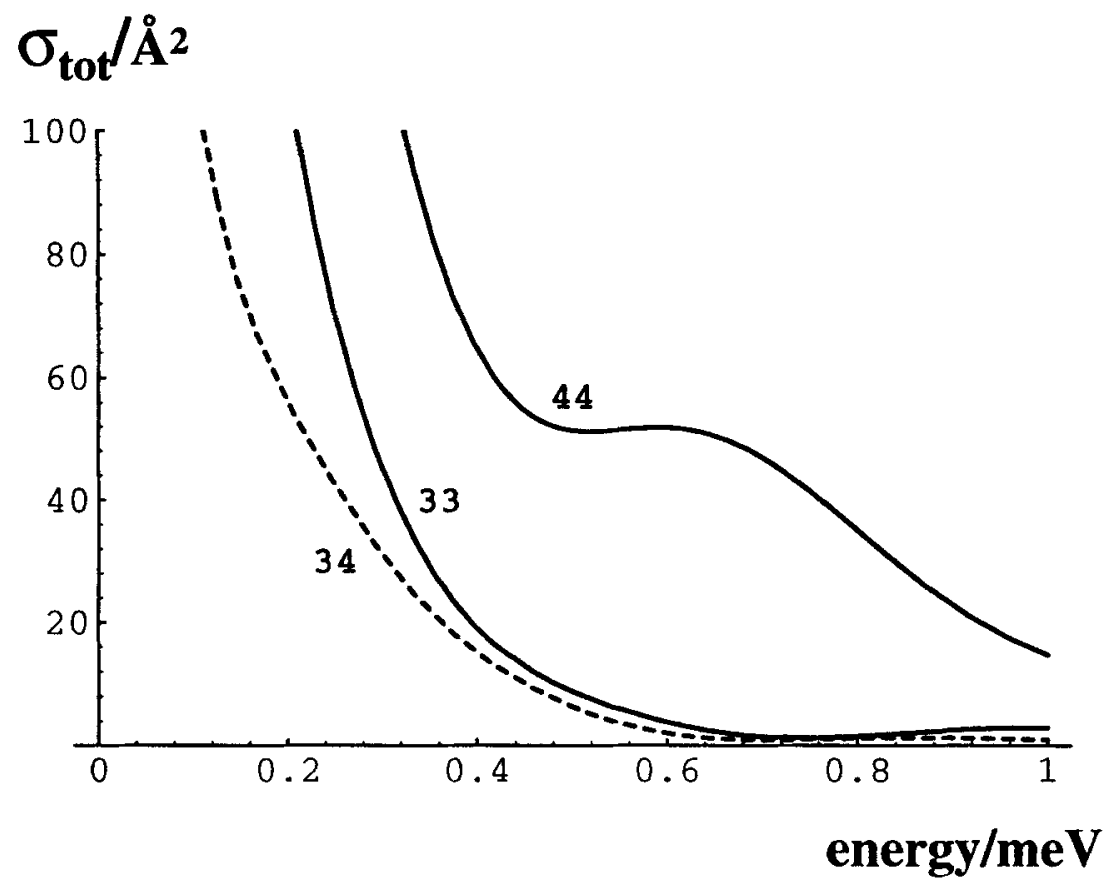

FIGURE 3. Total cross section vs. center-of-mass energy for He-He scattering. The three isotopic combinations; 4-4, 3-3, and 3-4 (dashed) are shown.

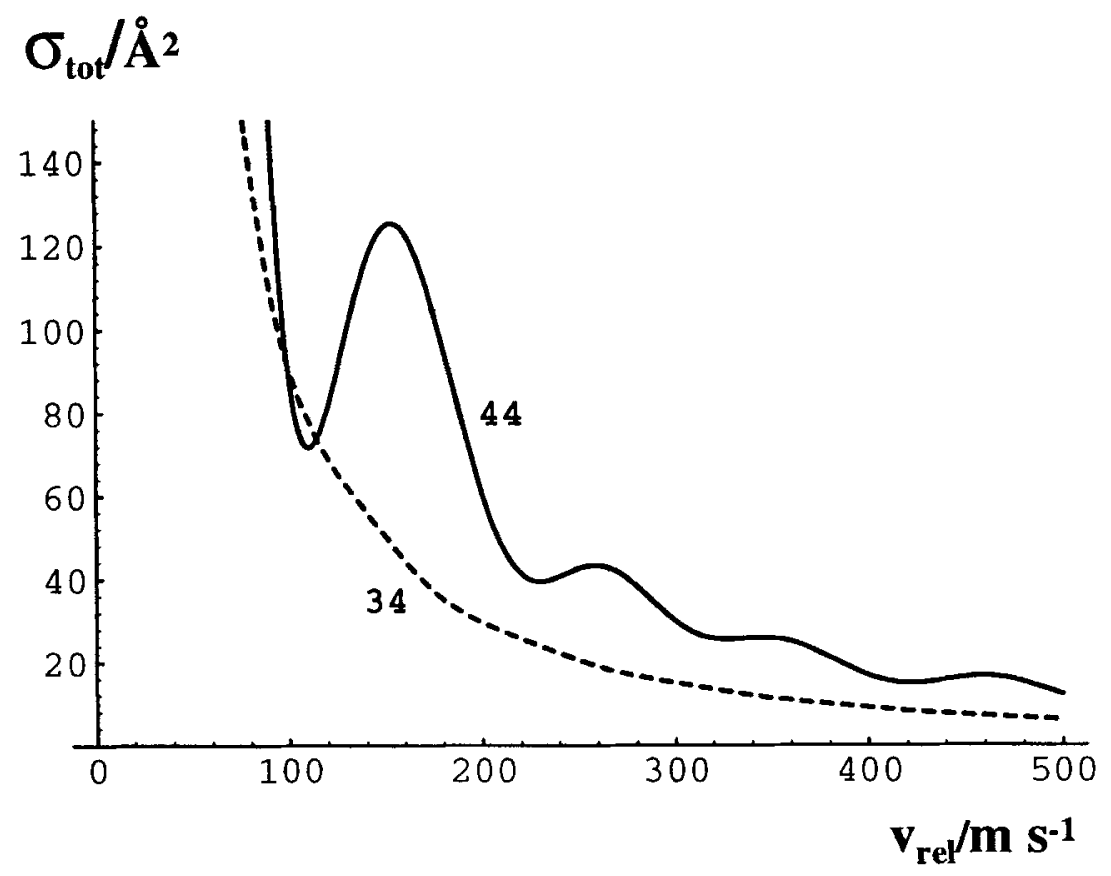

FIGURE 4. Total cross section vs. relative beam velocity. Glory scattering can be seen for the helium 4-4 case. 
The interference term in (24) involves the integral

$$
\begin{gathered}
\int_{0}^{\pi} \frac{\sin \left(K r_{0}\right)}{K} \frac{\sin \left(K^{\prime} r_{0}\right)}{K^{\prime}} \sin \theta d \theta, \\
K^{\prime} \equiv 2 k \cos \frac{\theta}{2} .
\end{gathered}
$$

This works out to

$$
\begin{array}{r}
\frac{1}{2 k^{2}} \int_{0}^{\pi} \sin \left(2 k r_{0} \sin \frac{\theta}{2}\right) \sin \left(2 k r_{0} \cos \frac{\theta}{2}\right) d \theta \\
=2 r_{0}^{2} G\left(2 k r_{0}\right)
\end{array}
$$

in terms of a rapidly convergent sum over Bessel functions of odd order:

$$
\begin{aligned}
G(x) & \equiv \sum_{n, m=0}^{\infty} C_{n m} J_{2 n+1}(x) J_{2 m+1}(x) \\
C_{n m} & =\frac{(4 m+2)(-)^{n}-(4 n+2)(-)^{m}}{(m-n)(m+n+1)}, \\
C_{n n} & =\frac{4(-)^{n}}{2 n+1} .
\end{aligned}
$$

The total scattering cross section is

$$
\sigma_{\text {tot }}^{4-4}=8 \pi r_{0}^{2} \lambda^{2}\left[F\left(2 k r_{0}\right)+G\left(2 k r_{0}\right)\right] .
$$

For helium 3-3 scattering, the analogous result is

$$
\sigma_{\text {tot }}^{3-3}=8 \pi r_{0}^{2} \lambda^{2}\left[F\left(2 k r_{0}\right)-\frac{1}{2} G\left(2 k r_{0}\right)\right] .
$$

Figure 4 shows plots of $\sigma_{\text {tot }}$ vs. relative beam velocity. The oscillations in the helium $4-4$ cross section correspond to glory extrema observed in 4-4 scattering [13]. They also occur for helium 3-3 (not shown) but not for helium 3-4 and are thus correctly accounted for by our model.

\section{Summary}

We have demonstrated that a simple Dirac bubble potential model is remarkably capable of representing several distinctive features of the helium dimer: the existence of exactly one bound state for ${ }^{4} \mathrm{He}_{2}$, with $J=0$; the instability of its isotopic variants; the extreme delocalization of the vibrational wave function; the minute ratio of binding energy to well depth; the suppression of odd partial waves in ${ }^{4} \mathrm{He}-{ }^{4} \mathrm{He}$ scattering; and the occurrence of glory scattering. With minimal computational efforts, we have thus been able to reproduce results previously obtained only by elaborate ab initio computations. We anticipate that our model potential might find application for other noble gas interactions and in simulations involving clusters of three or more helium atoms.

\section{References}

1. F. Luo, G. C. McBane, G. Kim, C. F. Giese, and W. R. Gentry, J. Chem. Phys. 98, 3564 (1993)

2. R. A. Aziz and M. J. Slaman, J. Chem. Phys. 94, 8047 (1991).

3. R. A. Aziz and M. J. Slaman, Metrologia 27, 211 (1990).

4. B. Liu and A. D. McLean, J. Chem. Phys, 91, 2348 (1989).

5. R. J. Vos, J. H. van Lenthe and F. B. van Duijneveldt, J. Chem. Phys. 93, 643 (1990).

6. J. B. Anderson, C. A. Traynor, and B. M. Boghosian, J. Chem. Phys. 99, 345 (1993).

7. F. Luo, G. Kin, G. C. McBane, C. F. Giese, and W. R. Gentry, J. Chem. Phys. 98, 9687 (1993)

8. Y.-H. Uang and W.C. Stwalley, J. Chem. Phys. 76, 5069 (1982).

9. For other $\mathrm{He}_{2}$ calculations, see G. J. Tawa, P.A. Whitlock, J.W. Moscowitz, and K.W. Schmidt, Int. J. Supercomput. Appl. 5, 57 (1991); G. Chalasiński and M. Gutowski, Chem. Rev. 88, 943 (1988).

10. S. M. Blinder, Chem. Phys. Lett. 64, 485 (1979).

11. S. M. Blinder, Phys. Rev. A 18, 853 (1978).

12. L. L. Lohr and S. M. Blinder, Chem. Phys. Lett. 198, 100 (1992).

13. R. Feltgen, H. Kirst, K. A. Kohler, H. Pauly, and F. Torello, J. Chem. Phys. 76, 2360 (1982). 